\title{
On Supercurrent Superfields and Fayet-Iliopoulos Terms in $N=1$ Gauge Theories
}

\author{
Daniel Arnold ${ }^{1}$, Jean-Pierre Derendinger ${ }^{1}$ and Jelle Hartong ${ }^{2}$ \\ 1 Albert Einstein Center for Fundamental Physics, \\ Institute for Theoretical Physics, Bern University \\ Sidlerstrasse 5, CH-3012 Bern, Switzerland \\ 2 Niels Bohr Institute, Blegdamsvej 17, \\ DK-2100 Copenhagen Ø, Denmark
}

\begin{abstract}
We revisit the supermultiplet structure of Noether currents for $N=1$ supersymmetric gauge theories. Using superfield identities and the field equations we show how to derive a superfield equation for the divergences of the Noether currents in terms of the supercurrent and anomaly superfields containing $16_{B}+16_{F}$ components. We refer to this as the natural supercurrent structure as it is invariant under all local symmetries of the theory. It corresponds to the $\mathcal{S}$-multiplet of Komargodski and Seiberg. We clarify the on/off-shell nature of the currents appearing in this multiplet and we study in detail the effect of specific improvement transformations leading to 1) a Ferrara-Zumino multiplet and to 2) a multiplet containing the new improved energy-momentum tensor of Callan, Coleman and Jackiw. Our methods also apply to supersymmetric gauge theories with a Fayet-Iliopoulos term. We construct the natural supercurrent multiplet for such a theory and show how to improve this to a formally gauge-invariant Ferrara-Zumino multiplet by introducing a non-dynamical chiral superfield $S$ to ensure superfield gauge invariance. Finally we study the coupling of this theory to supergravity and show that $S$ remains non-dynamical if the theory is $R$-symmetric and that $S$ becomes propagating if the theory is not $R$-symmetric, leading to non-minimal $16_{B}+16_{F}$ supergravity.
\end{abstract}




\section{Introduction}

Any Poincaré-invariant $N=1$ supersymmetric theory has conserved supercurrent and energy-momentum tensor. In 1975, studying the Wess-Zumino model, Ferrara and Zumino found that these conserved Noether currents belong to a supermultiplet described by a real supercurrent superfield

$$
J_{\mu}=\left(\bar{\sigma}_{\mu}\right)^{\dot{\alpha} \alpha} J_{\alpha \dot{\alpha}}, \quad J_{\alpha \dot{\alpha}}=\frac{1}{2}\left(\sigma^{\mu}\right)_{\alpha \dot{\alpha}} J_{\mu}
$$

verifying a supercurrent superfield equation [1]. Since a symmetric energy-momentum tensor and the supercurrent include $10_{B}+16_{F}$ fields 1 , or $6_{B}+12_{F}$ fields with their conservation equations, the supercurrent superfield necessarily includes other objects matching bosons and fermions. Ferrara and Zumino also found that if $\bar{D}^{\dot{\alpha}} J_{\alpha \dot{\alpha}}=0$, the theory has conserved dilatation and chiral $R$-symmetry currents and is actually superconformal with $8_{B}+8_{F}$ operators: a symmetric, conserved and traceless energymomentum tensor $\partial^{\mu} T_{\mu \nu}=T_{\mu}^{\mu}=0$, a conserved $R$-symmetry current, $\partial^{\mu} j_{\mu}=0$, and a conserved supercurrent with zero "gamma trace", $\partial^{\mu} S_{\mu}=S_{\mu} \sigma^{\mu}=0$. One can show on general grounds that these properties hold for every supersymmetric field theory, by demanding for instance that the conserved Noether charges derived from the currents generate the supersymmetry algebra [2, 3. The argument however assumes that the Noether charges are well-defined operators.

A generic supersymmetric theory is not superconformal and the chiral and dilatation currents are then not conserved. Their violations find sources in so-called classical or quantum anomalies, which can also, under the same circumstances, be described by anomaly superfields in the supercurrent superfield equation. This superfield equation should be such that the super-Poincaré conservation laws $\partial^{\mu} T_{\mu \nu}=\partial^{\mu} S_{\mu}=0$ are maintained, but $\partial^{\mu} j_{\mu}, T^{\mu}{ }_{\mu}$, and $S_{\mu} \sigma^{\mu}$ may be nonzero.

In this work, we use the terminology supercurrent structure for the supercurrent superfield, the anomaly superfields and the corresponding supercurrent superfield equation.

An exhaustive study of supercurrent structures in $N=1$ theories is a subtle problem which is not our subject2. We consider in this article the already quite general situation where the supercurrent superfield equation is of the form

$$
\bar{D}^{\dot{\alpha}} J_{\alpha \dot{\alpha}}=\Delta_{\alpha}, \quad D^{\alpha} J_{\alpha \dot{\alpha}}=-\bar{\Delta}_{\dot{\alpha}}
$$

with the complex spinor superfield $\Delta_{\alpha}$ which describes anomalies involving two contri-

\footnotetext{
${ }^{1}$ We use this notation for the number of bosonic and fermionic off-shell components.

${ }^{2}$ See for instance refs. [4, 5, 6].
} 
butions 3

$$
\Delta_{\alpha}=D_{\alpha} X+\chi_{\alpha}, \quad \bar{\Delta}_{\dot{\alpha}}=-\bar{D}_{\dot{\alpha}} \bar{X}+\bar{\chi}_{\dot{\alpha}},
$$

where $X$ is a chiral superfield $\left(4_{B}+4_{F}\right)$. The superfield $\chi_{\alpha}$ is also chiral, it includes an antisymmetric tensor verifying the Bianchi identity $D^{\alpha} \chi_{\alpha}+\bar{D}_{\dot{\alpha}} \bar{\chi}^{\dot{\alpha}}=0$. Hence,

$$
\chi_{\alpha}=-\frac{1}{4} \overline{D D} D_{\alpha} U, \quad U^{\dagger}=U
$$

and $\chi_{\alpha}$ also includes $4_{B}+4_{F}$ fields 4 . In the supercurrent equation, $D_{\alpha} X$ and $\chi_{\alpha}$ describe respectively the chiral and linear anomalies.

For a given supersymmetric theory, one expects as a matter of principle to find an expression for the superfields $J_{\alpha \dot{\alpha}}, X$ and $\chi_{\alpha}$ in terms of the off-shell superfields of the theory. The use of superspace relies on linear supersymmetry, and then on off-shell fields. For on-shell fields solving the field equations, searching for superfield expressions for the currents does not make sense in general. The supercurrent equation, however, is a conservation equation which only holds for solutions of the field equations. This is a standard feature of the Noether prescription: currents can be directly calculated from the Lagrangian and the variational principle or the field equations provide then the (non-)conservation equations.

The second remark at this stage is that the supercurrent structure of a given theory is not unique. There are superfield identities which act on $J_{\alpha \dot{\alpha}}, X$ and $\chi_{\alpha}$ leaving the supercurrent equations (1.2) and (1.3) unchanged for the transformed superfields. Since identities do not contain significant, dynamical information, the transformation of the conserved $T_{\mu \nu}$ and $S_{\mu}$ is an improvement of these currents. But other operators of the supercurrent structure are transformed significantly. For instance, the lowest component of $J_{\mu}$ is the current of a $U(1)_{R}$ rotation of the fields in the theory which is significantly modified in the transformation. Similarly, the relation between the divergence of the dilatation current and the trace of the energy-momentum tensor changes in the transformation. We will give a detailed discussion of these transformations of a supercurrent structure.

In the next sections, we will proceed to give various expressions for $J_{\alpha \dot{\alpha}}$ for an arbitrary supersymmetric gauge theory with matter superfields. As indicated earlier, these expressions apply to off-shell superfields and are then unambiguous. Then, secondly, using the field equations of the theory, we will calculate the anomaly superfields $X$ and $\chi_{\alpha}$ and equation (1.2) will then contain all information on the conservation or violation of superconformal symmetries. This unambiguous procedure does work as

\footnotetext{
${ }^{3}$ For consistency, the anomaly superfield $\Delta_{\alpha}$ is linear, $\overline{D D} \Delta_{\alpha}=0$. It then includes at most $24_{B}+24_{F}$ components. But the conservation of the super-Poincaré currents reduces these numbers.

${ }^{4}$ Since $\chi_{\alpha}$ is invariant under $U \rightarrow U+\Lambda+\bar{\Lambda}$ with $\bar{D}_{\dot{\alpha}} \Lambda=0$
} 
easily for a theory with a Fayet-Iliopoulos term and, by construction, all expressions will be automatically gauge-invariant.

The standard methods to derive a supercurrent structure are either 5 to couple the theory to an off-shell (linearized) background supergravity and obtain the currents from the variation of the background fields, or the (superconformal) superfield Noether procedure of ref. [4]. However, we should not rely on a specific off-shell supergravity formulation since we use improvement transformations which relate different supergravity formulations and we are primarily interested in theories which are neither scale- nor $R$-invariant. We then use a more heuristic but simpler method.

Our $N=1$ superspace procedure is very similar to standard Noether currents and their (non-)conservation in a generic field theory. It is as simple-minded as this: consider any function $\mathcal{L}\left(\varphi, \partial_{\mu} \varphi\right)$ of a single real field. A (linear) variation of $\varphi$ leads to the straightforward identity

$$
\partial^{\mu}\left(\frac{\partial \mathcal{L}}{\partial \partial^{\mu} \varphi} \delta \varphi\right)=\delta \mathcal{L}-\left(\frac{\partial \mathcal{L}}{\partial \varphi}-\partial^{\mu} \frac{\partial \mathcal{L}}{\partial \partial^{\mu} \varphi}\right) \delta \varphi .
$$

Then, if $\mathcal{L}$ is the Lagrangian and $\varphi$ solves the field equation, identity (1.5) turns into a (non-)conservation equation for the Noether current

$$
j_{\mu}^{N}=\frac{\partial \mathcal{L}}{\partial \partial^{\mu} \varphi} \delta \varphi
$$

with source $\delta \mathcal{L}$. Notice however that the expression of the current can be derived from $\mathcal{L}$ in terms of the off-shell field. Similarly, for all supersymmetric theories, one can define $J_{\alpha \dot{\alpha}}$, expressed in terms of off-shell superfields, associated with a suitable identity which then generates the supercurrent equation, verified by on-shell fields only.

In Section 2, we briefly recall the field content of the supercurrent structure and equation, borrowing mostly from ref. [9], and we add some comments in preparation of the following sections.

Section 3 considers a generic supersymmetric gauge theory, in three steps: deriving superfield identities, obtaining a "natural" supercurrent structure from these identities and improving the natural structure. We discuss the nature of the energy-momentum tensor, its relation to the dilatation current, the nature of the $R$-current and the role of auxiliary fields. We also identify the improvement transformation which leads to the supercurrent structure that contains the new improved energy-momentum tensor of Callan, Coleman and Jackiw (CCJ) [10] and the Noether current of superconformal $U(1)_{R}$ transformations. This particular supercurrent structure will be of importance in

\footnotetext{
${ }^{5}$ As described for instance in the textbooks [7] and [8].
} 
an upcoming paper [11], in which we study the anomalies of $N=1$ super-Yang-Mills in relation to the NSVZ $\beta$ function using effective field theory techniques.

Section 4 discusses the supercurrent structure of abelian gauge theories with a FayetIliopoulos term, following the same steps as in section [3. The "natural" supercurrent structure has $16_{B}+16_{F}$ operators, but we also show how to obtain a gauge-invariant Ferrara-Zumino $12_{B}+12_{F}$ structure with the formal introduction of $4_{B}+4_{F}$ new fields in a chiral superfield $S$ without any dynamical content. We then discuss the corresponding construction in supergravity. We point out that the chiral multiplet $S$ naturally appears through a gauge transformation in the superconformal formulation of new minimal Poincaré supergravity. We then study the old minimal version and we show how a generic superpotential produces an obstruction which disappears in the global supersymmetry limit. The obstruction is evaded if, as expected, the superpotential is $R$-symmetric and the $R$-symmetry is gauged. With a generic superpotential, the way out is to turn $S$ into a dynamical supermultiplet, i.e. to couple the globally supersymmetric theory to non-minimal $16_{B}+16_{F}$ supergravity. Our results provide us with a new perspective on the coupling of a supersymmetric gauge theory with a FayetIliopoulos term to supergravity and complement the conclusions of ref. [9]. Conclusions and a technical appendix close the paper.

\section{The supercurrent structure}

In preparation for the next sections, we begin with a discussion of the solution of the supercurrent superfield equation. We mostly follow earlier literature and in particular ref. [9] but we also try to clarify and make precise several aspects which sometimes create confusion. Again, the equations to solve are

$$
\bar{D}^{\dot{\alpha}} J_{\alpha \dot{\alpha}}=D_{\alpha} X+\chi_{\alpha}, \quad \bar{D}_{\dot{\alpha}} X=0, \quad \chi_{\alpha}=-\frac{1}{4} \overline{D D} D_{\alpha} U, \quad U=U^{\dagger} .
$$

These equations for the supercurrent superfield are actually not the most general allowing conserved energy-momentum tensor and supercurrent [4, 5, 6], but they suffice for our purposes. In total, superfields $J_{\alpha \dot{\alpha}}, X$ and $\chi_{\alpha}$ include $40_{B}+40_{F}$ real components. Since the supercurrent superfield equation is complex linear (it vanishes identically under $\overline{D D})$, it imposes $2 \times\left(12_{B}+12_{F}\right)$ conditions on the $40_{B}+40_{F}$ components to leave a solution expressed in terms of $16_{B}+16_{F}$ fields.

The superfield identity

$$
2 \bar{D}^{\dot{\alpha}}\left[D_{\alpha}, \bar{D}_{\dot{\alpha}}\right] \mathcal{G}=D_{\alpha} \overline{D D} \mathcal{G}+3 \overline{D D} D_{\alpha} \mathcal{G}
$$


which holds for any superfield $\mathcal{G}$, can be used to transform the supercurrent structure into another solution of equations (2.1):

$$
\begin{array}{rll}
J_{\alpha \dot{\alpha}} & \longrightarrow & \widetilde{J}_{\alpha \dot{\alpha}}=J_{\alpha \dot{\alpha}}+2\left[D_{\alpha}, \bar{D}_{\dot{\alpha}}\right] \mathcal{G}, \\
X & \longrightarrow & \widetilde{X}=X+\overline{D D} \mathcal{G}, \\
\chi_{\alpha} & \longrightarrow & \tilde{\chi}_{\alpha}=\chi_{\alpha}+3 \overline{D D} D_{\alpha} \mathcal{G},
\end{array}
$$

with $\mathcal{G}$ real. Hence, each theory admits in principle a (continuous) family of supercurrent structures. Notice that if $\mathcal{G}$ is linear $(\overline{D D} \mathcal{G}=0), \widetilde{X}=X$. Similarly, if $\mathcal{G}=\Psi+\bar{\Psi}$, $\bar{D}_{\dot{\alpha}} \Psi=0$, then $\widetilde{\chi}_{\alpha}=\chi_{\alpha}$. But the use of transformations (2.3) may face various obstructions if conditions like gauge invariance or global definition are imposed on the supercurrent structure $J_{\alpha \dot{\alpha}}, X, \chi_{\alpha} 6$

There are three obvious reductions. If $\chi_{\alpha}=0$, or if $\chi_{\alpha}$ can be canceled using transformations (2.3), the resulting supercurrent structure has $12_{B}+12_{F}$ fields and chiral anomaly $X$. This is the original Ferrara-Zumino [1] structure. Transformations (2.3) can still be used with $\mathcal{G}=\Psi+\bar{\Psi}$. Similarly, if $X=0$, or if $X$ can be canceled using transformations (2.3), the supercurrent structure has again $12_{B}+12_{F}$ fields with linear anomaly $\chi_{\alpha}$. Transformations $(2.3)$ can still be used with a linear $\mathcal{G}$. Finally, if transformation (2.3) can be used to obtain a supercurrent with $X=\chi_{\alpha}=0$, it has $8_{B}+8_{F}$ fields and the theory is superconformal.

To solve in terms of component fields the supercurrent equation, we use the following expansion of the chiral superfields $X$ and $\chi_{\alpha}$ :

$$
\begin{aligned}
X(y, \theta) & =x+\sqrt{2} \theta \psi_{X}-\theta \theta f_{X}, \\
\chi_{\alpha}(y, \theta) & =-i \lambda_{\alpha}+\theta_{\alpha} D+\frac{i}{2}\left(\theta \sigma^{\mu} \bar{\sigma}^{\nu}\right)_{\alpha} F_{\mu \nu}-\theta \theta\left(\sigma^{\mu} \partial_{\mu} \bar{\lambda}\right)_{\alpha},
\end{aligned}
$$

in chiral coordinates 7 and with $F_{\mu \nu}=\partial_{\mu} U_{\nu}-\partial_{\nu} U_{\mu}$. For the superfield $U$, the last eq. (2.1) implies

$$
U=\theta \sigma^{\mu} \bar{\theta} U_{\mu}+i \theta \theta \overline{\theta \lambda}-i \overline{\theta \theta} \theta \lambda+\frac{1}{2} \theta \theta \overline{\theta \theta} D+\ldots
$$

where the dots denote components of $U$ absent from $\chi_{\alpha}$. The resulting supercurrent

\footnotetext{
${ }^{6}$ Although these superfields are not strictly speaking physical quantities. These conditions have been discussed in ref. [9] for specific theories. They will reappear in later sections.

${ }^{7}$ Appendix A gives complete formula.
} 
superfield can then be written 8

$$
\begin{aligned}
J_{\mu}(x, \theta, \bar{\theta})= & \frac{8}{3} j_{\mu}(x)+\theta\left(S_{\mu}+2 \sqrt{2} \sigma_{\mu} \bar{\psi}_{X}\right)+\bar{\theta}\left(\bar{S}_{\mu}-2 \sqrt{2} \bar{\sigma}_{\mu} \psi_{X}\right) \\
& -2 i \theta \theta \partial_{\mu} \bar{x}+2 i \overline{\theta \theta} \partial_{\mu} x \\
& +\theta \sigma^{\nu} \bar{\theta}\left[8 T_{\mu \nu}-4 \eta_{\mu \nu} \operatorname{Re} f_{X}-\frac{1}{2} \epsilon_{\mu \nu \rho \sigma}\left(\frac{8}{3} \partial^{\rho} j^{\sigma}-F^{\rho \sigma}\right)\right] \\
& -\frac{i}{2} \theta \theta \bar{\theta}\left(\partial_{\nu} S_{\mu} \sigma^{\nu}+2 \sqrt{2} \bar{\sigma}_{\mu} \sigma^{\nu} \partial_{\nu} \bar{\psi}_{X}\right) \\
& +\frac{i}{2} \overline{\theta \theta} \theta\left(\sigma^{\nu} \partial_{\nu} \bar{S}_{\mu}+2 \sqrt{2} \sigma_{\mu} \bar{\sigma}^{\nu} \partial_{\nu} \psi_{X}\right) \\
& -\frac{2}{3} \theta \theta \overline{\theta \theta}\left(2 \partial_{\mu} \partial^{\nu} j_{\nu}-\square j_{\mu}\right)
\end{aligned}
$$

with $T_{\mu \nu}=T_{\nu \mu}$. This expression solves eq. (2.1) if $T_{\mu \nu}$ and $S_{\mu}$ verify conservation equations

$$
\partial^{\mu} T_{\mu \nu}=0, \quad \partial^{\mu} S_{\mu}=0 .
$$

Hence, $T_{\mu \nu}$ and $S_{\mu}$ will be (proportional to) the conserved energy-momentum tensor and the supercurrent.

In addition, the supercurrent equation indicates that the following additional relations are verified:

$$
\begin{gathered}
4 T_{\mu}^{\mu}=D+6 \operatorname{Re} f_{X}, \quad \partial^{\mu} j_{\mu}=-\frac{3}{2} \operatorname{Im} f_{X} \\
\left(\sigma^{\mu} \bar{S}_{\mu}\right)_{\alpha}=6 \sqrt{2} \psi_{X \alpha}+2 i \lambda_{\alpha} .
\end{gathered}
$$

The first condition indicates that $D$ and $\operatorname{Re} f_{X}$ are sources for the trace of the energymomentum tensor. Its precise significance depends on the specific energy-momentum tensor included in $J_{\mu}$ : since $T_{\mu \nu}$ is defined up to improvements, is it not in general true that a scale-invariant theory has a traceless energy-momentum tensor. The second condition indicates that $\operatorname{Im} f_{X}$ induces the non-conservation of $j_{\mu}$, which is related in general to an $R$ transformation acting in the theory. The third condition controls the violation of superconformal supersymmetry.

For $X \neq 0 \neq \chi_{\alpha}$, the supercurrent superfield $J_{\mu}$ includes a conserved symmetric energy-momentum tensor $T_{\mu \nu}\left(10_{B}-4_{B}=6_{B}\right)$, the conserved supercurrent $S_{\mu}(4 \times$ $\left.(4-1)_{F}=12_{F}\right)$ and a vector current $j_{\mu}$ which is not conserved $\left(4_{B}\right)$. Since conditions (2.8) eliminate $2_{B}+4_{F}$, the source superfields $X$ and $\chi_{\alpha}$ add $6_{B}+4_{F}$ fields, for a total of $16_{B}+16_{F}$ fields.

\footnotetext{
${ }^{8}$ We do not define a normalization for the supercurrent $S_{\mu}$, we will not use its explicit expression.
} 
Denoting the components of $\mathcal{G}$ by $\left(C_{g}, \chi_{g}, v_{g \mu}, \ldots\right)$, the component fields $j_{\mu}, S_{\mu}$ and $T_{\mu \nu}$ of $J_{\mu}$ change into

$$
\begin{array}{rll}
j_{\mu} & \longrightarrow & \tilde{j}_{\mu}=j_{\mu}-3 v_{g \mu}, \\
S_{\mu} & \longrightarrow & \tilde{S}_{\mu}=S_{\mu}+8 \sigma_{[\mu} \bar{\sigma}_{\nu]} \partial^{\nu} \chi_{g}, \\
T_{\mu \nu} & \longrightarrow & \tilde{T}_{\mu \nu}=T_{\mu \nu}+\left(\partial_{\mu} \partial_{\nu}-\eta_{\mu \nu} \square\right) C_{g},
\end{array}
$$

under the transformation (2.3). Clearly, $S_{\mu}$ and $T_{\mu \nu}$ are changed by improvements, i.e. trivially conserved terms whose $\mu=0$ components are spatial derivatives leaving the corresponding Noether charges unaffected. But, unless $v_{g \mu}=\partial^{\nu} A_{\mu \nu}$ with some $A_{\mu \nu}=-A_{\nu \mu}$, the vector field $j_{\mu}$ which is not in general conserved is more significantly transformed into a completely different current. This new current could be associated, by Noether procedure, to a different global transformation of the fields in the theory.

Some remarks are in order. Firstly, notice that the components of the anomaly superfields $X$ and $\chi_{\alpha}$ appear in $J_{\mu}$. Hence, the symmetric part of the $\theta \sigma^{\nu} \bar{\theta}$ component of $J_{\mu}$ can only be identified with an energy-momentum tensor of the theory after subtraction of an anomaly contribution generated by $\operatorname{Re} f_{X}$, or by $D$, or by both, since we may as well use the first eq. (2.8) to modify the component expansion (2.6) 9

Secondly, even if, for a given theory, one expects to find expressions for $J_{\alpha \dot{\alpha}}, X$ and $\chi_{\alpha}$ in terms of superfields, i.e. in terms of off-shell fields, equations (2.6) $-(2.8)$ only hold for on-shell fields. The interpretation of the components of $J_{\mu}$ in terms of currents may require the field equations. This is in particular true, as we will see later on, for the auxiliary field contributions.

\section{Supersymmetric gauge theory}

In this section, we consider an arbitrary $N=1$ gauge theory with matter superfields $\phi^{i}$ in some representation of the gauge group. In most expressions however, we will use the notation $\Phi$ to denote the collection of all chiral superfields $\phi^{i}$, viewed as a column matrix, and eliminate indices. Except otherwise indicated, the gauge vector superfield $A$ is valued in this representation: $A=A^{a} T^{a}$, with generators $T^{a}$ in the representation of $\Phi$. Gauge transformations have a chiral parameter $\Lambda=\Lambda^{a} T^{a}, \bar{D}_{\dot{\alpha}} \Lambda=0$. They read:

$$
\begin{aligned}
& \Phi \quad \longrightarrow \quad e^{\Lambda} \Phi, \quad \bar{\Phi} \quad \longrightarrow \quad \bar{\Phi} e^{\bar{\Lambda}}, \\
& e^{A} \quad \longrightarrow \quad e^{-\bar{\Lambda}} e^{A} e^{-\Lambda}, \quad e^{-A} \quad \longrightarrow \quad e^{\Lambda} e^{-A} e^{\bar{\Lambda}}
\end{aligned}
$$

\footnotetext{
${ }^{9}$ The omission of these anomaly contributions seems to be at the origin of the erroneous no-go statement of ref. [12, as observed in ref. 9].
} 
and $\bar{\Phi} e^{A} \Phi$ is gauge-invariant. The gauge covariant supersymmetric derivatives are

$$
\mathcal{D}_{\alpha} \Phi=e^{-A} D_{\alpha}\left(e^{A} \Phi\right), \quad \overline{\mathcal{D}}_{\dot{\alpha}} \bar{\Phi}=\bar{D}_{\dot{\alpha}}\left(\bar{\Phi} e^{A}\right) e^{-A}
$$

and $\left(\overline{\mathcal{D}}_{\dot{\alpha}} \bar{\Phi}\right) e^{A}\left(\mathcal{D}_{\alpha} \Phi\right)$ is then also gauge-invariant 10

\subsection{An identity for matter superfields}

We begin our discussion of the supercurrent with a superfield identity. For any real function $K\left(\bar{\Phi} e^{A} \Phi\right)$, some simple manipulations lead to

$$
2 \bar{D}^{\dot{\alpha}}\left[\left(\overline{\mathcal{D}}_{\dot{\alpha}} \bar{\Phi}\right) K_{\Phi \Phi}\left(\mathcal{D}_{\alpha} \Phi\right)\right]=-\overline{D D} D_{\alpha} K-4 K_{\Phi} \mathcal{W}_{\alpha} \Phi-\left(\overline{D D} K_{\Phi}\right)\left(\mathcal{D}_{\alpha} \Phi\right)
$$

where

$$
\mathcal{W}_{\alpha}=-\frac{1}{4} \overline{D D} e^{-A} D_{\alpha} e^{A}, \quad \quad \overline{\mathcal{W}}_{\dot{\alpha}}=\frac{1}{4} D D e^{A} \bar{D}_{\dot{\alpha}} e^{-A}
$$

are the non-abelian field strength superfields11 with gauge transformations

$$
\mathcal{W}_{\alpha} \quad \longrightarrow \quad e^{\Lambda} \mathcal{W}_{\alpha} e^{-\Lambda}, \quad \overline{\mathcal{W}}_{\dot{\alpha}} \longrightarrow e^{-\bar{\Lambda}} \overline{\mathcal{W}}_{\dot{\alpha}} e^{\bar{\Lambda}}
$$

The notation

$$
K_{\Phi}=\frac{\partial K}{\partial \Phi}, \quad K_{\bar{\Phi}}=\frac{\partial K}{\partial \bar{\Phi}}, \quad K_{\Phi \bar{\Phi}}=\frac{\partial^{2} K}{\partial \Phi \partial \bar{\Phi}}
$$

is used. We stress that the gauge-invariant eq. (3.3) is an identity, it does not contain any information. It will be used to define the supercurrent superfield $J_{\alpha \dot{\alpha}}$ of a WessZumino model with Kähler potential $K$, up to improvements to be discussed later on.

Notice that we use for simplicity the gauge-invariant variable $\bar{\Phi} e^{A} \Phi$. But formula (3.3) actually holds for an arbitrary gauge-invariant function $K$. We will also assume that the theory does not include gauge-singlet chiral superfields. Then, if the Kähler potential depends on real gauge-invariant variables like $\bar{\Phi} e^{A} \Phi$, it always has a global $($ non- $R) U(1)$ symmetry.

\subsection{An identity for gauge superfields}

We may derive a similar identity for gauge superfields. The tool is the non-abelian Bianchi identity

$$
e^{-A} D^{\alpha}\left(e^{A} \mathcal{W}_{\alpha} e^{-A}\right) e^{A}=\bar{D}_{\dot{\alpha}}\left(e^{-A} \overline{\mathcal{W}}^{\dot{\alpha}} e^{A}\right)
$$

\footnotetext{
${ }^{10}$ As usual, $D_{\alpha}=\frac{\partial}{\partial \theta^{\alpha}}-i\left(\sigma^{\mu} \bar{\theta}\right)_{\alpha} \partial_{\mu}$ and $\bar{D}_{\dot{\alpha}}=\frac{\partial}{\partial \bar{\theta}^{\alpha}}-i\left(\theta \sigma^{\mu}\right)_{\dot{\alpha}} \partial_{\mu}$.

${ }^{11}$ Notice that with these standard but somewhat unfortunate definitions, $\overline{\mathcal{W}}_{\dot{\alpha}}=-\left(\mathcal{W}_{\alpha}\right)^{\dagger}$.
} 
Multiplying (left) by $\mathcal{W}_{\alpha}$ and taking the trace gives

$$
\bar{D}^{\dot{\alpha}} \operatorname{Tr}\left[\mathcal{W}_{\alpha} e^{-A} \overline{\mathcal{W}}_{\dot{\alpha}} e^{A}\right]=\operatorname{Tr}\left[e^{A} \mathcal{W}_{\alpha} e^{-A} D^{\beta}\left(e^{A} \mathcal{W}_{\beta} e^{-A}\right)\right]
$$

Then, for an arbitrary (gauge-invariant) holomorphic function $g(\Phi)$,

$$
\begin{aligned}
\bar{D}^{\dot{\alpha}}\left[(g+\bar{g}) \operatorname{Tr}\left[\mathcal{W}_{\alpha} e^{-A} \overline{\mathcal{W}}_{\dot{\alpha}} e^{A}\right]\right]= & (g+\bar{g}) \operatorname{Tr}\left[e^{A} \mathcal{W}_{\alpha} e^{-A} D^{\beta}\left(e^{A} \mathcal{W}_{\beta} e^{-A}\right)\right] \\
& +\left(\bar{D}^{\dot{\alpha}} \bar{g}\right) \operatorname{Tr}\left[\mathcal{W}_{\alpha} e^{-A} \overline{\mathcal{W}}_{\dot{\alpha}} e^{A}\right]
\end{aligned}
$$

Identities (3.3) and (3.9) are the building blocks of the supercurrent structure for the gauge-invariant Wess-Zumino model, which we consider next.

\subsection{The theory and its supercurrent structures}

We consider the following gauge-invariant Wess-Zumino model:

$$
\mathcal{L}=\int d^{2} \theta d^{2} \bar{\theta} K\left(\bar{\Phi} e^{A} \Phi\right)+\int d^{2} \theta\left[W(\Phi)+\frac{1}{4} g(\Phi) \widetilde{\operatorname{Tr}}\left(\mathcal{W}^{\alpha} \mathcal{W}_{\alpha}\right)\right]+\text { h.c. }
$$

The holomorphic functions $W$ and $g$ are assumed invariant under the non-abelian supersymmetric gauge transformations (3.1). Gauge kinetic terms are normalized using $\widetilde{\operatorname{Tr}}=\frac{1}{T(R)} \operatorname{Tr}, \operatorname{Tr}\left(T^{a} T^{b}\right)=T(R) \delta^{a b}$. Notice that with our choice of variable, since 12

$$
K_{\Phi} \Phi=\bar{\Phi} K_{\bar{\Phi}}=K^{\prime} \bar{\Phi} e^{A} \Phi
$$

the Kähler potential part of the theory is always invariant under the non- $R U(1)$ symmetry rotating all chiral superfields $\phi^{i}$ by the same phase. In general, a non-trivial gauge kinetic function $g$ or a superpotential $W$ will break this symmetry. Assuming scale dimension $w$ for all chiral superfield 13 , scale invariance of the Kähler potential part of $\mathcal{L}$ corresponds then to the condition $w K_{\Phi} \Phi=K$ and

$$
\Delta=2\left(K-w K_{\Phi} \Phi\right)
$$

measures the violation of scale invariance. Similarly, the holomorphic quantity

$$
\widetilde{\Delta}=3 W-w W_{\Phi} \Phi
$$

\footnotetext{
${ }^{13}$ Our conventions for scale transformations are

$$
\begin{array}{rll}
x^{\mu} & \longrightarrow & e^{-\lambda} x^{\mu}, \\
\theta & \longrightarrow & e^{-\lambda / 2} \theta, \\
\Phi(x) & \longrightarrow & e^{w \lambda} \Phi\left(e^{\lambda} x\right) .
\end{array}
$$
}

${ }^{12}$ The prime denotes the first derivative of $K$ with respect to its variable $\bar{\Phi} e^{A} \Phi$. 
measures the violation of scale invariance in the superpotential terms. Actually, if $j_{\mu}^{(d i l .)}$ is the Noether current for dilatations, we have on-shell 14

$$
\begin{aligned}
\partial^{\mu} j_{\mu}^{(d i l .)}= & 2(w-1) K_{z \bar{z}}\left[\left(\partial^{\mu} \bar{z}\right)\left(\partial_{\mu} z\right)+\bar{f} f\right]-(w-3)\left[W_{z} f+\overline{f W_{\bar{z}}}\right] \\
& +2 w K_{\bar{z} z z} z\left[\left(\partial^{\mu} \bar{z}\right)\left(\partial_{\mu} z\right)+\bar{f} f\right]-w W_{z z} z f-w \overline{W_{\overline{z z}}} \bar{z} \bar{f} \\
& + \text { fermions }+ \text { gauge terms } \\
= & -\left.\Delta\right|_{\theta \theta \overline{\theta \theta}}-\left.\widetilde{\Delta}\right|_{\theta \theta}-\left.\overline{\widetilde{\Delta}}\right|_{\overline{\theta \theta}}-\left.\frac{1}{4} \square \Delta\right|_{\theta=0}+\text { gauge terms }
\end{aligned}
$$

and the gauge terms are proportional to $w g_{z} z$ or $w \overline{z g} \bar{z}_{\bar{z}}$. Hence scale invariance holds if

$$
\Delta=\widetilde{\Delta}=w g_{z} z=0 .
$$

The Lagrangian induces field equations

$$
\overline{D D} K_{\Phi}=4 W_{\Phi}+g_{\Phi} \widetilde{\operatorname{Tr}}\left(\mathcal{W}^{\alpha} \mathcal{W}_{\alpha}\right)
$$

for the chiral superfield $\Phi$ and, for $A$,

$$
(g+\bar{g}) D^{\alpha}\left(e^{A} \mathcal{W}_{\alpha} e^{-A}\right)=2 T(R) K^{\prime} e^{A} \Phi \bar{\Phi}-\left(D^{\alpha} g\right) e^{A} \mathcal{W}_{\alpha} e^{-A}-\left(\bar{D}_{\dot{\alpha}} \bar{g}\right) \overline{\mathcal{W}}^{\dot{\alpha}} .
$$

The Bianchi identity (3.7) has been used to simplify the field equations for $A$.

Next, we insert the field equations into our identities. The resulting equations then hold only for on-shell fields. Using the first identity (3.3) and matter field equation (3.16), one obtains

$$
-2 \bar{D}^{\dot{\alpha}}\left[\left(\overline{\mathcal{D}}_{\dot{\alpha}} \bar{\Phi}\right) K_{\Phi \Phi}\left(\mathcal{D}_{\alpha} \Phi\right)\right]=\overline{D D} D_{\alpha} K+4 D_{\alpha} W+4 K_{\Phi} \mathcal{W}_{\alpha} \Phi+\widetilde{\operatorname{Tr}}\left(\mathcal{W}^{\beta} \mathcal{W}_{\beta}\right) D_{\alpha} g .
$$

Using the second identity (3.9) and the gauge field equation (3.17), one also gets

$$
-2 \bar{D}^{\dot{\alpha}}\left[(g+\bar{g}) \widetilde{\operatorname{Tr}}\left(\mathcal{W}_{\alpha} e^{-A} \overline{\mathcal{W}}_{\dot{\alpha}} e^{A}\right)\right]=-4 K_{\Phi} \mathcal{W}_{\alpha} \Phi-\widetilde{\operatorname{Tr}}\left(\mathcal{W}^{\beta} \mathcal{W}_{\beta}\right) D_{\alpha} g
$$

Comparing, one finds the following supercurrent structure:

$$
\begin{aligned}
\bar{D}^{\dot{\alpha}} J_{\alpha \dot{\alpha}} & =D_{\alpha} X+\chi_{\alpha}, \\
J_{\alpha \dot{\alpha}} & =-2\left(\overline{\mathcal{D}}_{\dot{\alpha}} \bar{\Phi}\right) K_{\Phi \bar{\Phi}}\left(\mathcal{D}_{\alpha} \Phi\right)-2(g+\bar{g}) \widetilde{\operatorname{Tr}}\left(\mathcal{W}_{\alpha} e^{-A} \overline{\mathcal{W}}_{\dot{\alpha}} e^{A}\right), \\
X & =4 W \\
\chi_{\alpha} & =\overline{D D} D_{\alpha} K .
\end{aligned}
$$

One may view these equations as the natural supercurrent structure for the gauged Wess-Zumino model. It uses the full $16_{B}+16_{F}$ structure 15 All quantities are gaugeinvariant and also invariant under Kähler transformations $K \rightarrow K+\Xi(\Phi)+\Xi(\bar{\Phi})$. And,

\footnotetext{
${ }^{14}$ We use the expansion $\Phi=z+\sqrt{2} \theta \psi-\theta \theta f$. Furthermore, since $K_{\Phi} \Phi=\bar{\Phi} K_{\bar{\Phi}}, z K_{z z \bar{z}}=\bar{z} K_{\overline{z z} z}$.

${ }^{15}$ This corresponds to the $\mathcal{S}$-multiplet of ref. [9].
} 
as we discuss below, the energy-momentum tensor included in $J_{\alpha \dot{\alpha}}$ is the canonical (Noether) tensor, improved to the symmetric gauge-invariant (Belinfante) tensor 16. The improvement transformation (2.3) can in principle be used to reduce the structure, but a nonzero superpotential is not in general of the form $-\overline{D D} \mathcal{G}$ and $X$ cannot then be removed. There are exceptions. For instance, if the superpotential is homogeneous, $W_{\Phi} \Phi=\ell W$, and if $g_{\Phi} \Phi=0$, the field equation implies $X=4 W=\ell^{-1} \overline{D D} K_{\Phi} \Phi$. Canceling $\chi_{\alpha}$ is always possible, at the price however of losing Kähler invariance of the transformed $J_{\alpha \dot{\alpha}}$ and $X$, with consequences explained in ref. [9]. Notice also that while the chiral anomaly term $D_{\alpha} X$ is generated by the field equation for $\Phi$, the linear anomaly $\chi_{\alpha}$ is truly off-shell: it is already present in the identity (3.3).

Theory (3.10) has kinetic metrics $\frac{1}{2}(g+\bar{g}) \delta_{a b}$ for the gauge multiplet and $K_{z \bar{z}}$ for the chiral superfield components. The lowest component of $J_{\alpha \dot{\alpha}}$ is the fermionic current 17

$$
\begin{aligned}
\left.j_{\mu} \equiv \frac{3}{8}\left(\bar{\sigma}_{\mu}\right)^{\dot{\alpha} \alpha} J_{\alpha \dot{\alpha}}\right|_{\theta=0} & =\frac{3}{2} \bar{\psi} K_{z \bar{z}} \bar{\sigma}_{\mu} \psi-\frac{3}{4}(g+\bar{g}) \bar{\lambda} \bar{\sigma}_{\mu} \lambda \\
& =\frac{3}{4}\left[\bar{\psi} K_{z \bar{z}} \gamma_{\mu} \gamma_{5} \psi-\frac{1}{2}(g+\bar{g}) \bar{\lambda} \gamma_{\mu} \gamma_{5} \lambda\right] .
\end{aligned}
$$

This is the current of chiral $U(1)$ rotations of the (two-component) fermion fields with charges $3 / 2$ for the gaugino (we choose this normalization for $R$-transformations of gauginos and Grassmann superspace coordinates) and $-3 / 2$ for chiral fermions. Hence, $j_{\mu}$ is the current of the $U(1)_{\widetilde{R}}$ group leaving chiral superfields inert, which is a symmetry for all Kähler potentials $K$ and gauge kinetic functions $g$ if the superpotential (and then $X$ ) vanishes 18 Since however $\chi_{\alpha}$ is never zero, the energy-momentum tensor included in $J_{\alpha \dot{\alpha}}$ is never traceless, even if the theory is scale-invariant.

In the expansion (2.6) of the supercurrent superfield (3.20) we find the following bosonic energy-momentum tensor:

$$
\begin{aligned}
T_{\mu \nu}= & \left(D_{\mu} \bar{z}\right) K_{z \bar{z}}\left(D_{\nu} z\right)+\left(D_{\nu} \bar{z}\right) K_{z \bar{z}}\left(D_{\mu} z\right)-\frac{1}{2}(g+\bar{g}) F_{\mu \rho}^{a} F_{\nu}^{a \rho} \\
& -\eta_{\mu \nu}\left[\left(D_{\rho} \bar{z}\right) K_{z \bar{z}}\left(D^{\rho} z\right)-\frac{1}{8}(g+\bar{g}) F_{\rho \sigma}^{a} F^{a \rho \sigma}+\bar{f} K_{z \bar{z}} f-\frac{1}{4}(g+\bar{g}) d^{a} d^{a}\right] \\
& +\frac{1}{2} \eta_{\mu \nu} \operatorname{Re} f_{X},
\end{aligned}
$$

\footnotetext{
${ }^{16}$ Strictly speaking, the difference between the Belinfante and the canonical (Noether) energymomentum tensors is not an improvement. It uses the field equations for gauge fields.

${ }^{17}$ We use the notation $\lambda$ for gauginos.

18 For a generic $R$-symmetry, transformations are
}

$$
\begin{array}{rll}
\theta & \longrightarrow & e^{3 i \alpha / 2} \theta, \\
\mathcal{W}_{\alpha} & \longrightarrow & e^{3 i \alpha / 2} \mathcal{W}_{\alpha} \\
\Phi & \longrightarrow & e^{i q \alpha} \Phi
\end{array}
$$

The case $q=0$ corresponds to $U(1)_{\widetilde{R}}$ transformations. 
where

$$
\begin{aligned}
D_{\mu} z & =\partial_{\mu} z+\frac{i}{2} T^{a} z A_{\mu}^{a}, \\
F_{\mu \nu}^{a} & =\partial_{\mu} A_{\nu}^{a}-\partial_{\nu} A_{\mu}^{a}-\frac{1}{2} f^{a b c} A_{\mu}^{b} A_{\nu}^{c}
\end{aligned}
$$

are the gauge-covariant derivatives of the scalars and the gauge-field strength tensor respectively. Since $X=4 W, \operatorname{Re} f_{X}=2\left(W_{z} f+\bar{f}_{\bar{z}}\right)$. As already stated, besides auxiliary field contributions which we discuss below, $T_{\mu \nu}$ is the canonical (Noether) tensor, improved to the symmetric gauge-invariant (Belinfante) tensor.

The auxiliary field structure is interesting. The bosonic contributions involving $f$ (in $\Phi=-\theta \theta f+\ldots$ ) and $d^{a}$ (in $A^{a}=\frac{1}{2} \theta \theta \overline{\theta \theta} d^{a}+\ldots$ ) are:

$$
\begin{array}{rlrl} 
& \text { In } T_{\mu \nu}: & T_{\mu \nu}^{(\text {aux. })} & =-\eta_{\mu \nu}\left[\bar{f} K_{z \bar{z}} f-W_{z} f-\overline{f W_{\bar{z}}}-\frac{1}{4}(g+\bar{g}) d^{a} d^{a}\right] . \\
& f_{X}^{(\text {aux. })} & =4 W_{z} f . \\
& D^{(a u x .)} & =-4 K_{z} T^{a} z d^{a}-8 \bar{f} K_{z \bar{z}} f . \\
& \text { In } \chi_{\alpha}: & T_{\mu}^{\mu(a u x .)} & =-K_{z} T^{a} z d^{a}-2 \bar{f} K_{z \bar{z}} f+3\left(W_{z} f+\bar{f} W_{\bar{z}}\right), \\
& \text { In eqs. (2.8): } & \partial^{\mu} j_{\mu}^{(a u x .)} & =-6 \operatorname{Im}\left(W_{z} f\right) .
\end{array}
$$

If the energy-momentum tensor were expressed as a function of off-shell auxiliary fields, the Noether procedure would lead to

$$
T_{\mu \nu}^{(a u x .)}=-\eta_{\mu \nu} \mathcal{L}^{(a u x .)}=-\eta_{\mu \nu}\left[\bar{f} K_{z \bar{z}} f-W_{z} f-\overline{f W}_{\bar{z}}+\frac{1}{4}(g+\bar{g}) d^{a} d^{a}+\frac{1}{2} d^{a} K_{z} T^{a} z\right] .
$$

It turns out that the component expansion (2.6) provides the off-shell expression for chiral superfields, but not for gauge fields. One can check that if $X \neq 0 \neq \chi_{\alpha}$, the auxiliary field energy-momentum tensor included in $J_{\alpha \dot{\alpha}}$ is never completely "off-shell", essentially because the component expansion of $J_{\alpha \dot{\alpha}}$ follows from the supercurrent equation which holds on-shell. Using the first eq. (2.8) does not help. Instead, replacing auxiliary fields by their on-shell values,

$$
K_{z \bar{z}} f=\bar{W}_{\bar{z}}, \quad(g+\bar{g}) d^{a}=-K_{z} T^{a} z,
$$

we find then $T_{\mu \nu}^{(a u x .)}=\eta_{\mu \nu} \mathcal{V}$ with scalar potential

$$
\mathcal{V}=\bar{f} K_{z \bar{z}} f+\frac{g+\bar{g}}{4} d^{a} d^{a}=W_{z}\left(K_{z \bar{z}}\right)^{-1} \bar{W}_{\bar{z}}+\frac{1}{4(g+\bar{g})} \sum_{a}\left(K_{z} T^{a} z\right)^{2},
$$

and all equations in (3.24) are of course consistent with the anomaly relations (2.8). Hence, the correct identification of the energy-momentum tensor, even if $J_{\alpha \dot{\alpha}}$ is expressed in terms of off-shell superfields, holds on-shell only. Since

$$
\widetilde{F}_{\mu \rho}^{a} F_{\nu}^{a \rho}-\frac{1}{4} \eta_{\mu \nu} \widetilde{F}_{\rho \sigma}^{a} F^{a \rho \sigma} \equiv 0
$$


$\left(\widetilde{F}_{\mu \nu}^{a}=\frac{1}{2} \epsilon_{\mu \nu \rho \sigma} F^{a \rho \sigma}\right)$ the energy-momentum tensor in $J_{\alpha \dot{\alpha}}$ [eq. (3.22)] does not depend on $\widetilde{F}_{\mu \nu}^{a}$ and then on $\operatorname{Im} g(z)$.

Theory (3.10) is expected to be scale-invariant if $\Delta=\widetilde{\Delta}=w g_{z} z=0$ [eq. (3.15)] ]. Since however $\chi_{\alpha} \neq 0$ in the supercurrent structure (3.20), the energy-momentum tensor (3.22) is never traceless, even if the theory is scale-invariant. In the canonical formulation leading to energy-momentum tensor (3.22) the dilatation current $j_{\mu}^{(d i l .)}$ verifies

$$
j_{\mu}^{(d i l .)}=x^{\nu} T_{\mu \nu}^{(\text {can. })}+\sum_{i} w_{i} \frac{\partial \mathcal{L}}{\partial \partial^{\mu} \varphi_{i}} \varphi_{i},
$$

where $\varphi_{i}$ is a generic field with scale dimension $w_{i}$ and $T_{\mu \nu}^{(\text {can. })}=\sum_{i} \frac{\partial \mathcal{L}}{\partial \partial^{\mu} \varphi_{i}} \partial_{\nu} \varphi_{i}-\eta_{\mu \nu} \mathcal{L}$ is the canonical energy-momentum tensor. Hence,

$$
T_{\mu}^{\left(\text {can. } \mu_{\mu}\right.}=\partial^{\mu} j_{\mu}^{(d i l .)}-\sum_{i} w_{i} \partial^{\mu}\left(\frac{\partial \mathcal{L}}{\partial \partial^{\mu} \varphi_{i}} \varphi_{i}\right),
$$

which is nonzero if $\partial^{\mu} j_{\mu}^{(d i l .)}=0$.

Callan, Coleman and Jackiw (CCJ) [10]19 have demonstrated how to improve the energy-momentum tensor into a new expression $\Theta_{\mu \nu}=\Theta_{\nu \mu}$ which verifies

$$
j_{\mu}^{(d i l .)}=x^{\nu} \Theta_{\mu \nu}, \quad \partial^{\mu} j_{\mu}^{(d i l .)}=\Theta_{\mu}^{\mu},
$$

and is on-shell traceless if the theory is scale-invariant. We now want to discuss two different supersymmetric improvements of the "natural" supercurrent structure (3.20) leading either to a Ferrara-Zumino structure with $\chi_{\alpha}=0$ or to the CCJ energymomentum tensor.

\subsection{Improvement to the Ferrara-Zumino supercurrent}

The supersymmetric improvement transformation (2.3) is generated by a real superfield $\mathcal{G}$. We first choose

$$
\mathcal{G}=-\frac{1}{3} K
$$

to eliminate $\chi_{\alpha}$ in the supercurrent structure (3.20). The result is the commonly used $12_{B}+12_{F}$ Ferrara-Zumino structure

$$
\begin{aligned}
\bar{D}^{\dot{\alpha}} J_{\alpha \dot{\alpha}}^{(1)} & =D_{\alpha} X^{(1)} \\
J_{\alpha \dot{\alpha}}^{(1)} & =-2\left(\overline{\mathcal{D}}_{\dot{\alpha}} \bar{\Phi}\right) K_{\Phi \bar{\Phi}}\left(\mathcal{D}_{\alpha} \Phi\right)-2(g+\bar{g}) \widetilde{\operatorname{Tr}}\left(\mathcal{W}_{\alpha} e^{-A} \overline{\mathcal{W}}_{\dot{\alpha}} e^{A}\right)-\frac{2}{3}\left[D_{\alpha}, \bar{D}_{\dot{\alpha}}\right] K \\
X^{(1)} & =4 W-\frac{1}{3} \overline{D D} K .
\end{aligned}
$$

\footnotetext{
${ }^{19}$ See for instance ref. [13, section 2.4 , for a general discussion.
} 
Since $X^{(1)}$ and $J_{\alpha \dot{\alpha}}^{(1)}$ are not invariant under Kähler transformations, an obstruction to this transformation could arise if the global description of the sigma-model target space requires patches with Kähler potentials linked by Kähler transformations [9].

Using field equation (3.16), the chiral anomaly superfield $X^{(1)}$ can be written as

$$
X^{(1)}=\frac{4}{3} \widetilde{\Delta}-\frac{1}{6} \overline{D D} \Delta-\frac{w}{3} g_{\Phi} \Phi \widetilde{\operatorname{Tr}}\left(\mathcal{W}^{\alpha} \mathcal{W}_{\alpha}\right)
$$

It vanishes in a scale-invariant theory where $\Delta=\widetilde{\Delta}=w g_{\Phi} \Phi=0$. Since $\chi_{\alpha}^{(1)}=0$, we have

$$
T_{\mu}^{(1) \mu}=\frac{3}{2} \operatorname{Re} f_{X^{(1)}}
$$

and the energy-momentum tensor in $J_{\alpha \dot{\alpha}}^{(1)}$ is traceless in a scale-invariant theory where $\partial^{\mu} j_{\mu}^{(d i l .)}=0$. However, for a generic theory without scale invariance, we have

$$
T_{\mu}^{(1) \mu}=\frac{3}{2} \operatorname{Re} f_{X^{(1)}} \neq \partial^{\mu} j_{\mu}^{(d i l .)} \neq 0 .
$$

The difference between the "natural" energy-momentum tensor $T_{\mu \nu}$ [eq. (3.22)] and the improved version is

$$
T_{\mu \nu}^{(1)}-T_{\mu \nu}=-\frac{1}{3}\left(\partial_{\mu} \partial_{\nu}-\eta_{\mu \nu} \square\right) K
$$

which is not the improvement to the tensor $\Theta_{\mu \nu}$ found by CCJ.

For clarity of the next equations, we now use the notation

$$
K_{i}=K_{z^{i}}=\frac{\partial K}{\partial z^{i}}, \quad K^{i}=K_{\bar{z}_{i}}=\frac{\partial K}{\partial \bar{z}_{i}}, \quad \ldots
$$

The lowest component of the supercurrent superfield $J_{\alpha \dot{\alpha}}^{(1)}$ then reads

$$
j_{\mu}^{(1)}=\frac{1}{2} \sum_{i, j} K_{i}^{j} \bar{\psi}_{j} \bar{\sigma}_{\mu} \psi^{i}+i \sum_{i}\left(K^{i} D_{\mu} \bar{z}_{i}-K_{i} D_{\mu} z^{i}\right)-\frac{3}{4}(g+\bar{g}) \bar{\lambda} \bar{\sigma}_{\mu} \lambda .
$$

This current has the following origin. Suppose that we start with a superconformal gauge theory, as in the superconformal construction of the $N=1$ supergravity-matter system [14]. We have then an auxiliary vector field $A_{\mu}$ to gauge the $U(1)_{R}$ symmetry inside $S U(2,2 \mid 1) \supset S U(2,2) \times U(1)_{R}$. The field equation for this auxiliary field is then 20

$$
A_{\mu} \sim j_{\mu}^{(1)}
$$

Even if the fermionic terms seem correct, the current $j_{\mu}^{(1)}$ is not the Noether current of the $R$-symmetry with canonical weights 1 for chiral superfields and $3 / 2$ for gauginos. For the chiral superfield terms, it should be viewed as the Kähler connection. This

\footnotetext{
${ }^{20}$ See ref. [15], eq. (33).
} 
chiral current is not in general conserved, except if $f_{X^{(1)}}=0$, i.e. if the theory has conformal symmetry.

Hence, this improved supercurrent structure with $12_{B}+12_{F}$ fields does not include particularly attractive currents, in obvious relation with the potential symmetries of the globally supersymmetric gauge theory. But it may be useful in the coupling to (linearized) supergravity, using the old minimal $12_{B}+12_{F}$ supergravity off-shell multiplet.

\subsection{Improvement to the CCJ supercurrent structure}

Suppose instead that we choose

$$
\mathcal{G}=-\frac{1}{6} w\left(K_{\Phi} \Phi+\bar{\Phi} K_{\bar{\Phi}}\right)=-\frac{1}{3} w K_{\Phi} \Phi
$$

The number $w$ will be, as earlier, identified with the scale weight of all chiral superfields. The resulting supercurrent structure is

$$
\begin{aligned}
\bar{D}^{\dot{\alpha}} J_{\alpha \dot{\alpha}}^{(2)} & =D_{\alpha} X^{(2)}+\chi_{\alpha}^{(2)} \\
J_{\alpha \dot{\alpha}}^{(2)} & =-2\left(\overline{\mathcal{D}}_{\dot{\alpha}} \bar{\Phi}\right) K_{\Phi \bar{\Phi}}\left(\mathcal{D}_{\alpha} \Phi\right)-2(g+\bar{g}) \widetilde{\operatorname{Tr}}\left(\mathcal{W}_{\alpha} e^{-A} \overline{\mathcal{W}}_{\dot{\alpha}} e^{A}\right)-\frac{2}{3} w\left[D_{\alpha}, \bar{D}_{\dot{\alpha}}\right] K_{\Phi} \Phi \\
X^{(2)} & =4 W-\frac{w}{3}\left(\overline{D D} K_{\Phi}\right) \Phi \\
\chi_{\alpha}^{(2)} & =\overline{D D} D_{\alpha}\left(K-w K_{\Phi} \Phi\right)=\frac{1}{2} \overline{D D} D_{\alpha} \Delta .
\end{aligned}
$$

The improved supercurrent superfield $J_{\alpha \dot{\alpha}}^{(2)}$ is not Kähler-invariant.

The first reason to consider this particular structure is that the lowest component of $J_{\alpha \dot{\alpha}}$ reads

$$
\begin{aligned}
j_{\mu}^{(2)}= & i w \sum_{i, j} K_{i}^{j}\left(z^{i} D_{\mu} \bar{z}_{j}-\bar{z}_{j} D_{\mu} z^{i}\right)-\frac{1}{2} w \sum_{i, j, k} \bar{\psi}_{j} \bar{\sigma}_{\mu} \psi^{i}\left(z^{k} K_{k i}^{j}+\bar{z}_{k} K_{i}^{k j}\right) \\
& -\left(w-\frac{3}{2}\right) \sum_{i, j} K_{i}^{j} \bar{\psi}_{j} \bar{\sigma}_{\mu} \psi^{i}-\frac{3}{4}(g+\bar{g}) \bar{\lambda} \bar{\sigma}_{\mu} \lambda .
\end{aligned}
$$

This is precisely the Noether current of the $R$ transformation, under which chiral superfields have $R$-charge $q=w$. On-shell, the superfield $X^{(2)}$ reads

$$
X^{(2)}=\frac{4}{3} \widetilde{\Delta}-\frac{w}{3} g_{\Phi} \Phi \widetilde{\operatorname{Tr}}\left(\mathcal{W}^{\alpha} \mathcal{W}_{\alpha}\right)
$$

Since we assume that $K_{\Phi} \Phi=\bar{\Phi} K_{\bar{\Phi}}$, the Kähler part of the theory is always $R$-invariant, for all $q$. Hence, $X^{(2)}$, which measures $\partial^{\mu} j_{\mu}^{(2)}$ should not, as in eq. (3.34), depend on $K$. $R$-symmetry follows if $\widetilde{\Delta}=w g_{\Phi} \Phi=0$, i.e. if $X^{(2)}$ vanishes. And as usual, scale invariance requires $\Delta=\widetilde{\Delta}=w g_{\Phi} \Phi=0$, implying $R$-invariance. 
The second reason to consider this supercurrent structure is that with this improvement, the superfield $J_{\alpha \dot{\alpha}}^{(2)}$ contains the CCJ energy-momentum tensor. One can actually verify that

$$
T_{\mu}^{(2) \mu}=\frac{3}{2} \operatorname{Re} f_{X^{(2)}}+\frac{1}{4} D^{(2)}=\partial^{\mu} j_{\mu}^{(d i l .)}
$$

in general, even if the theory is not scale-invariant.

Hence, this second improvement leads to a supercurrent structure with energymomentum tensor

$$
T_{\mu \nu}^{(2)}=\Theta_{\mu \nu}, \quad \Theta^{\mu}{ }_{\mu}=\partial^{\mu} j_{\mu}^{(d i l .)},
$$

and the Noether current of the $R$ transformation under which chiral superfields have charge $q=w$.

Finally, if $\Delta=0$ (and then $\chi_{\alpha}^{(2)}=0$ ), i.e. if $K=w K_{\Phi} \Phi$, the Kähler potential part of the theory is scale-invariant, and both improvements are of course identical. But they significantly differ in a generic theory.

\section{The Fayet-Iliopoulos term}

We now consider the case of super-Maxwell theory with a Fayet-Iliopoulos term, where a (non-R) $U(1)$ symmetry is gauged. This model has been considered by several author 21 , but we wish to present a somewhat different and unorthodox approach. The theory includes chiral superfields $\phi_{i}$ with $U(1)$ charges $q_{i}$ (we label both $\phi_{i}$ and $\bar{\phi}_{i}$ with the same lower index here) and gauge transformations

$$
\phi_{i} \quad \longrightarrow \quad e^{q_{i} \Lambda} \phi_{i}, \quad \bar{D}_{\dot{\alpha}} \Lambda=0
$$

and an abelian gauge superfield $A$ with $\delta A=-\Lambda-\bar{\Lambda}$. Gauge-covariant supersymmetric derivatives are

$$
\begin{aligned}
& \mathcal{D}_{\alpha} \phi_{i}=e^{-q_{i} A} D_{\alpha} e^{q_{i} A} \phi_{i}=D_{\alpha} \phi_{i}+q_{i}\left(D_{\alpha} A\right) \phi_{i}, \\
& \overline{\mathcal{D}}_{\dot{\alpha}} \bar{\phi}_{i}=e^{-q_{i} A} \bar{D}_{\dot{\alpha}} e^{q_{i} A} \bar{\phi}_{i}=\bar{D}_{\dot{\alpha}} \bar{\phi}_{i}+q_{i}\left(\bar{D}_{\dot{\alpha}} A\right) \bar{\phi}_{i}
\end{aligned}
$$

and identity (3.3) becomes

$$
2 \bar{D}^{\dot{\alpha}} \sum_{i, j}\left[K_{\phi_{i} \bar{\phi}_{j}}\left(\mathcal{D}_{\alpha} \phi_{i}\right)\left(\overline{\mathcal{D}}_{\dot{\alpha}} \bar{\phi}_{j}\right)\right]=\overline{D D} D_{\alpha} K+4 \sum_{i} q_{i} K_{\phi_{i}} \phi_{i} \mathcal{W}_{\alpha}+\sum_{i}\left(\overline{D D} K_{\phi_{i}}\right)\left(\mathcal{D}_{\alpha} \phi_{i}\right)
$$

Using Lagrangian

$$
\mathcal{L}=\int d^{2} \theta d^{2} \bar{\theta}\left[K\left(\bar{\phi}_{i} e^{q_{i} A} \phi_{i}\right)+\xi A\right]+\int d^{2} \theta\left[W\left(\phi_{i}\right)+\frac{1}{4} g\left(\phi_{i}\right) \mathcal{W}^{\alpha} \mathcal{W}_{\alpha}\right]+\text { h.c. }
$$

\footnotetext{
${ }^{21}$ See for instance [16, 17, 18, for recent discussions.
} 
where $\xi$ is the (dimension-two) Fayet-Iliopoulos (FI) coefficient and

$$
\mathcal{W}_{\alpha}=-\frac{1}{4} \overline{D D} D_{\alpha} A, \quad \overline{\mathcal{W}}_{\dot{\alpha}}=-\frac{1}{4} D D \bar{D}_{\dot{\alpha}} A,
$$

the field equations are

$$
\begin{aligned}
\overline{D D} K_{\phi_{i}} & =4 W_{\phi_{i}}+g_{\phi_{i}} \mathcal{W}^{\alpha} \mathcal{W}_{\alpha}, \\
\bar{D}_{\dot{\alpha}}\left[(g+\bar{g}) \overline{\mathcal{W}}^{\dot{\alpha}}\right] & =2 \xi+2 \sum_{i} q_{i} X_{i} \frac{\partial K}{\partial X_{i}}-\left(D^{\alpha} g\right) \mathcal{W}_{\alpha}, \quad X_{i}=\bar{\phi}_{i} e^{q_{i} A} \phi_{i} .
\end{aligned}
$$

The abelian Bianchi identity $D^{\alpha} \mathcal{W}_{\alpha}=\bar{D}_{\dot{\alpha}} \overline{\mathcal{W}}^{\dot{\alpha}}$ has been used. The two functions $W\left(\phi_{i}\right)$ and $g\left(\phi_{i}\right)$ are $U(1)$-invariant: $\sum_{i} q_{i} \phi_{i} W_{\phi_{i}}=\sum_{i} q_{i} \phi_{i} g_{\phi_{i}}=0$. Then, the first field equation also implies $\overline{D D} \sum_{i} q_{i} \phi_{i} K_{\phi_{i}}=0$.

The real Lagrangian superfield $K+\xi A$ is not invariant under supersymmetric gauge transformations. 22 But the non-invariance of the Lagrangian is confined to a derivative which does not generate dynamics and vanishes in the Wess-Zumino gauge. Using the expansion

$$
A=C+\theta \sigma^{\mu} \bar{\theta} A_{\mu}+\theta \theta \overline{\theta \theta}\left[\frac{1}{2} d-\frac{1}{4} \square C\right]+\ldots,
$$

$C$ transforms and vanishes in Wess-Zumino gauge, $d$ is gauge-invariant and we may use

$$
\mathcal{L}_{F I}=\xi\left[\frac{1}{2} d-\frac{1}{4} \square C\right]
$$

as the FI Lagrangian. Field equations (4.6) are then invariant or covariant under supersymmetric gauge transformations. Hence, the absence of gauge invariance of the Lagrangian superfield is irrelevant to the Lagrangian and to the dynamical equations. However, in the canonical formalism, a derivative contribution does induce, in particular, an energy-momentum tensor which does not affect the total energy-momentum of the theory, which is the physically significant quantity 23

In the supercurrent structure, one insists in working with superfields and then one will plausibly face gauge non-invariance of operators which are however not physical quantities. This is not so for the natural supercurrent structure. As in the non-abelian case, field equations imply the gauge-invariant equations

$$
\begin{aligned}
\sum_{i}\left(\overline{D D} K_{\phi_{i}}\right)\left(\mathcal{D}_{\alpha} \phi_{i}\right) & =4 D_{\alpha} W+\left(D_{\alpha} g\right) \mathcal{W}^{\beta} \mathcal{W}_{\beta} \\
\bar{D}^{\dot{\alpha}}\left[(g+\bar{g}) \mathcal{W}_{\alpha} \overline{\mathcal{W}}_{\dot{\alpha}}\right] & =2 \xi \mathcal{W}_{\alpha}+2 \sum_{i} q_{i} X_{i} \frac{\partial K}{\partial X_{i}} \mathcal{W}_{\alpha}+\frac{1}{2}\left(D_{\alpha} g\right) \mathcal{W}^{\beta} \mathcal{W}_{\beta}
\end{aligned}
$$

\footnotetext{
${ }^{22}$ Or Kähler transformations.

${ }^{23}$ In other words, it adds an improvement term to the physically relevant energy-momentum tensor.
} 
For fields solving field equations, identity (4.3) and the abelian version of formula (3.9) turn then into the "natural" $16_{B}+16_{F}$ supercurrent structure

$$
\begin{aligned}
\bar{D}^{\dot{\alpha}} J_{\alpha \dot{\alpha}} & =D_{\alpha} X+\chi_{\alpha}, \\
J_{\alpha \dot{\alpha}} & =2 \sum_{i, j} K_{\phi_{i} \bar{\phi}_{j}}\left(\mathcal{D}_{\alpha} \phi_{i}\right)\left(\overline{\mathcal{D}}_{\dot{\alpha}} \bar{\phi}_{j}\right)-2(g+\bar{g}) \mathcal{W}_{\alpha} \overline{\mathcal{W}}_{\dot{\alpha}} \\
X & =4 W \\
\chi_{\alpha} & =\overline{D D} D_{\alpha} K-4 \xi \mathcal{W}_{\alpha}=\overline{D D} D_{\alpha}(K+\xi A) .
\end{aligned}
$$

The Fayet-Iliopoulos coefficient appears, as it should, as a source of scale invariance breaking in $\chi_{\alpha}$. Its contribution is as expected gauge-invariant 24 The energymomentum tensor included in $J_{\alpha \dot{\alpha}}$ depends on $\xi$, according to the first condition (2.8), but only via the on-shell value of the auxiliary field $d .25$

\subsection{The improved supercurrent structure}

To obtain the supercurrent superfield which contains the new improved energy-momentum tensor $\Theta_{\mu \nu}$ and the appropriate $U(1)_{R}$ current, we now transform the supercurrent structure (4.9) with the real superfield $\mathcal{G}=-w \sum_{i} \phi_{i} K_{\phi_{i}} / 3$, w being as usual the (common) scale dimension of the chiral superfields. This leads to the supercurrent structure

$$
\begin{aligned}
\widetilde{J}_{\alpha \dot{\alpha}} & =2 \sum_{i, j} K_{\phi_{i} \bar{\phi}_{j}}\left(\mathcal{D}_{\alpha} \phi_{i}\right)\left(\overline{\mathcal{D}}_{\dot{\alpha}} \bar{\phi}_{j}\right)-\frac{2 w}{3}\left[D_{\alpha}, \bar{D}_{\dot{\alpha}}\right] \sum_{i} \phi_{i} K_{\phi_{i}}-2(g+\bar{g}) \mathcal{W}_{\alpha} \overline{\mathcal{W}}_{\dot{\alpha}} \\
\widetilde{X} & =4 W-\frac{w}{3} \sum_{i} \phi_{i} \overline{D D} K_{\phi_{i}} \\
\tilde{\chi}_{\alpha} & =\frac{1}{2} \overline{D D} D_{\alpha} \Delta-4 \xi \mathcal{W}_{\alpha} .
\end{aligned}
$$

As in the non-abelian case, using the first of field equations (4.6), $\widetilde{X}$ can be written as

$$
\widetilde{X}=\frac{4}{3} \widetilde{\Delta}-\frac{w}{3} \sum_{i} \phi_{i} g_{\phi_{i}} \mathcal{W}^{\alpha} \mathcal{W}_{\alpha}
$$

and the Kähler potential only contributes to scale symmetry violation in $\chi_{\alpha}$. The condition $\widetilde{\Delta}=0$ on the superpotential is necessary for $U(1)_{R}$ invariance of the theory. If in addition $\sum_{i} \phi_{i} g_{\phi_{i}}=0$, then $\widetilde{X}=0$ and the lowest component of $\widetilde{J}_{\alpha \dot{\alpha}}$ is conserved, as it should. Finally, the dimensionful Fayet-Iliopoulos term always breaks scale invariance without affecting the $R$-symmetry.

\footnotetext{
${ }^{24}$ For super-Maxwell theory $\left(\phi_{i}=0\right)$, this is the supercurrent structure obtained by Kuzenko [16].

${ }^{25}$ See the discussion following eqs. (3.24).
} 


\subsection{On the Ferrara-Zumino supercurrent structure with a Fayet-Iliopoulos term}

Our goal is to remove, using an improvement transformation (2.3), the contribution $\chi_{\alpha}=-4 \xi \mathcal{W}_{\alpha}=\xi \overline{D D} D_{\alpha} A$. But we want a gauge-invariant procedure: we cannot use $\mathcal{G}=-\xi A / 3$ in improvement transformation (2.3). But, as we observed earlier, the problem with gauge invariance is related to our prejudice to work with superfields and to the related presence of a derivative term in the FI Lagrangian. We then first make this derivative term gauge-invariant: we formally introduce a new chiral superfield $S$ and postulate that the quantity $A+S+\bar{S}$ is invariant under supersymmetric gauge transformations. Since

$$
\overline{D D} D_{\alpha}(A+S+\bar{S})=-4 \mathcal{W}_{\alpha}
$$

we prefer to consider the theory

$$
\mathcal{L}=\int d^{2} \theta d^{2} \bar{\theta}\left[K\left(\bar{\phi}_{i} e^{q_{i} A} \phi_{i}\right)+\xi(A+S+\bar{S})\right]+\int d^{2} \theta\left[W\left(\phi_{i}\right)+\frac{1}{4} g\left(\phi_{i}\right) \mathcal{W}^{\alpha} \mathcal{W}_{\alpha}\right]+\text { h.c. }
$$

with a gauge-invariant Lagrangian superfield. With expansion

$$
A+S+\bar{S}=C+2 \operatorname{Re} s+\ldots+\frac{1}{2} \theta \theta \overline{\theta \theta}\left[d-\frac{1}{2} \square(C+2 \operatorname{Re} s)\right]
$$

we find

$$
\xi \int d^{2} \theta d^{2} \bar{\theta}[A+S+\bar{S}]=\xi\left[\frac{1}{2} d-\frac{1}{4} \square(C+2 \operatorname{Re} s)\right] .
$$

Since $S$ does not generate any dynamict 26 , we are considering the same Fayet-Iliopoulos term as in theory (4.4), but defined now in terms of a real gauge-invariant superfield $A+S+\bar{S}$. Notice that the derivative terms in the Lagrangian will generate the following contribution to the canonical energy-momentum tensor:

$$
T_{\mu \nu}^{(S)}=-\frac{\xi}{4}\left(\partial_{\mu} \partial_{\nu}-\eta_{\mu \nu} \square\right)(C+2 \operatorname{Re} s), \quad T_{\mu}^{(S) \mu}=\frac{3}{4} \xi \square(C+2 \operatorname{Re} s) .
$$

But this is an improvement term that will not affect the Noether charges. Since the natural supercurrent structure (4.9) is unaffected by the introduction of $S$, it does not contain the improvement term (4.15). Notice also that a Kähler transformation generates an improvement contribution similar to eq. (4.15). Hence it can be used to eliminate the field $S$, confirming the formal character of its introduction.

We may then use the gauge-invariant superfield

$$
\mathcal{G}=-\frac{1}{3}[K+\xi(A+S+\bar{S})]
$$

\footnotetext{
${ }^{26}$ This is true in all gauges.
} 
in improvement transformation (2.3), to obtain the Ferrara-Zumino supercurrent structure

$$
\begin{array}{r}
\widehat{J}_{\alpha \dot{\alpha}}=2 \sum_{i, j} K_{\phi_{i} \bar{\phi}_{j}}\left(\mathcal{D}_{\alpha} \phi_{i}\right)\left(\overline{\mathcal{D}}_{\dot{\alpha}} \bar{\phi}_{j}\right)-2(g+\bar{g}) \mathcal{W}_{\alpha} \overline{\mathcal{W}}_{\dot{\alpha}} \\
-\frac{2}{3}\left[D_{\alpha}, \bar{D}_{\dot{\alpha}}\right][K+\xi(A+S+\bar{S})], \\
\widehat{X}=4 W-\frac{1}{3} \overline{D D}[K+\xi(A+S+\bar{S})]
\end{array}
$$

with $\widehat{\chi}_{\alpha}=0$. All quantities are gauge-invariant. The lowest component of the supercurrent superfield (4.17) reads

$$
\widehat{j}_{\mu}=j_{\mu}^{(1)}+\xi\left[A_{\mu}+2 \partial_{\mu} \operatorname{Im} s\right]
$$

with $j_{\mu}^{(1)}$ given in eq. (3.39). We also have that

$$
\operatorname{Im} f_{\widehat{X}}=\operatorname{Im} f_{X^{(1)}}-\frac{2}{3} \xi \partial^{\mu}\left[A_{\mu}+2 \partial_{\mu} \operatorname{Im} s\right]
$$

and then the equation

$$
\partial^{\mu} \widehat{j}_{\mu}=-\frac{3}{2} \operatorname{Im} f_{\widehat{X}}
$$

is just the equation for the (non-)conservation of $j_{\mu}^{(1)}$. Similarly, the energy-momentum tensor in supercurrent superfield (4.17) is

$$
\widehat{T}_{\mu \nu}=T_{\mu \nu}^{(1)}-\frac{\xi}{3}\left(\partial_{\mu} \partial_{\nu}-\eta_{\mu \nu} \square\right)(C+2 \operatorname{Re} s) .
$$

Since

$$
\operatorname{Re} f_{\widehat{X}}=\operatorname{Re} f_{X^{(1)}}-\frac{2}{3} \xi[d-\square(C+2 \operatorname{Re} s)]
$$

the equation

$$
\widehat{T}_{\mu}^{\mu}=\frac{3}{2} \operatorname{Re} f_{\widehat{X}}
$$

is equivalent to

$$
T_{\mu}^{(1) \mu}=\frac{3}{2} \operatorname{Re} f_{X^{(1)}}-\xi d
$$

and simply determines the trace of $T_{\mu \nu}^{(1)}$ as in eq. (3.35), but in the presence of the FayetIliopoulos term. All physically significant equations are unchanged by the introduction of $S$, but all quantities are now formally gauge-invariant.

Hence, we have needed $4_{B}+4_{F}$ new degrees of freedom to obtain the gauge-invariant Ferrara-Zumino structure (4.17). But the introduction of $S$ is purely formal, as is the problem of the gauge variation of the superfield FI term. It does not alter the 
dynamics of the theory nor does it change its symmetry properties. The new fields do not propagate 27

From the point of view of global supersymmetry, this is a fully satisfactory formulation of the supercurrent structure with a FI term. The introduction of the $S$ field, which as earlier observed can also be viewed as a particular Kähler transformation, is independent from the form of the superpotential: Kähler invariance of the globally supersymmetric theory does not involve the superpotential. Supergravity is different, it can be expressed in terms of the function $\mathcal{G}=K+\ln (W \bar{W})$, and a Kähler transformation must be compensated by a superpotential transformation.

\subsection{On the Fayet-Iliopoulos term in supergravity}

We now wish to uplift the construction of the previous section to supergravity. Our goal is to show how does the introduction of the chiral superfield $S$ survive and is actually naturally included in the supergravity coupling. We use the superconformal formulation of Poincaré supergravity, with a compensating supermultiplet to gauge fix superconformal symmetries absent in the super-Poincaré algebra. In the minimal sets of supergravity auxiliary fields, the compensating multiplet is either chiral (old minimal) or real linear (new minimal). We begin with the new minimal construction.

In the superconformal formulation, new minimal supergravity has Lagrangian

$$
\mathcal{L}_{n . m .}=\frac{3}{2}\left[L \ln \left(\frac{L}{S_{0} \bar{S}_{0}}\right)-L\right]_{D},
$$

where $L$ is the compensating real linear multiplet (Weyl weight $w=2$ ), $S_{0}$ is chiral with Weyl and chiral weights $w=n=1$ and $[\ldots]_{D}$ denotes the invariant $D$-density formula 28 . Since $[L(\Lambda+\bar{\Lambda})]_{D}$ is a derivative for any chiral $\Lambda, S_{0}$ contributes to the Lagrangian by a derivative only: it does not generate any field equation and its role is only to give the correct dimension to the argument of the logarithm. The theory has the obvious gauge invariance

$$
S_{0} \quad \longrightarrow \quad e^{-\Lambda^{\prime}} S_{0}
$$

which allows to choose a gauge where $S_{0}$ is a nonzero dimensionful constant. Tensor

\footnotetext{
${ }^{27}$ Using a non-dynamical superfield to restore a superspace local symmetry has been introduced in section 6 of ref. [19, to gauge symmetries leaving the Kähler potential invariant up to a Kähler transformation.

${ }^{28}$ We refer to Kugo and Uehara [15] for the superconformal calculus. We (almost) use their conventions and notations.
} 
calculus then shows that 29

$$
e^{-1} \mathcal{L}_{n . m .}=-\frac{1}{2} C_{L} R-\frac{3}{2} \ln \left(\frac{C_{L}}{z_{0} \bar{z}_{0}}\right) \square C_{L}+\ldots
$$

and the gauge-fixing of dilatation symmetry corresponds to

$$
C_{L}=\kappa^{-2} \equiv \frac{M_{P}^{2}}{8 \pi}
$$

in the Einstein frame.

We can then couple new minimal supergravity to gauge and matter supermultiplets. Assuming vanishing Weyl weights for the latter, the compensating multiplet $L$ is used to obtain the required Weyl weight $w=2$ in $D$-densities while $S_{0}$ can be used in the superpotential $F$-density provided the gauge invariance (4.26) is preserved. Consider then the theory

$$
\begin{aligned}
\mathcal{L}_{n . m .}= & \frac{3}{2}\left[L \ln \left(\frac{L}{S_{0} \bar{S}_{0}}\right)-L+\frac{1}{3} L K\left(\bar{\phi}_{i} e^{q_{i} A} \phi_{i}\right)+\frac{2}{3} \hat{\xi} L(A+S+\bar{S})\right]_{D} \\
& +\left[S_{0}^{3} W\left(\phi_{i}\right)+\frac{1}{4} g\left(\phi_{i}\right) \mathcal{W} \mathcal{W}\right]_{F} .
\end{aligned}
$$

There are two distinct cases. Firstly, if the superpotential does not vanish, gauge transformation (4.26) must then be compensated by a transformation of matter superfields

$$
\phi_{i} \longrightarrow e^{Q_{i} \Lambda^{\prime}} \phi_{i}
$$

with invariance conditions $W\left(e^{Q_{i} \Lambda^{\prime}} \phi_{i}\right)=e^{3 \Lambda^{\prime}} W\left(\phi_{i}\right)$ and $g\left(e^{Q_{i} \Lambda^{\prime}} \phi_{i}\right)=g\left(\phi_{i}\right)$. It is an $R$-symmetry acting on the $\phi_{i}$ and invariance of the Kähler potential $K$ implies then that $A$ gauges this $R$-symmetry, up to, maybe, a non- $R$ global symmetry. Notice that this condition is not related to the FI term: new minimal supergravity only admits $R$-symmetric superpotentials. Recall also that in the $R$-symmetric case, the natural supercurrent structure (4.9) of the globally supersymmetric theory has

$$
X=4 W=\overline{D D} \frac{1}{3} \sum_{i} Q_{i} \phi_{i} K_{\phi_{i}}
$$

using the first field equation (4.6). Hence, an improvement transformation (2.3) can cancel $X$ and we expect that the global theory admits a coupling to new minimal supergravity.

If the superpotential vanishes, gauge transformations of $S_{0}$ and $\phi_{i}$ remain decoupled. Since theory (4.29) only depends on $S_{0} \exp \left(-\frac{2}{3} \hat{\xi} S\right)$ in a derivative term, it is clear that

\footnotetext{
${ }^{29} C_{L}$ denotes the lowest component of the linear multiplet $L$.
} 
$S$ can either be produced from $S_{0}$ by a gauge transformation (4.26) or absorbed into $S_{0}$ by the inverse transformation. In any case, gauge invariances of $A$ and $S_{0}$ are preserved and independent. In the gauge $S=0$ however, gauge variations of $S_{0}$ and $A$ are identified, the quantity $\bar{S}_{0} e^{-\frac{2}{3} \hat{\xi} A} S_{0}$ is invariant: the FI coefficient is then related to the charge of $S_{0}$ under the transformation (4.26) gauged by $A$.

The old minimal formulation of theory (4.29) is obtained as follows. We first replace the linear $L$ by an unconstrained real vector superfield $U$ with Weyl weight $w=2$ :

$$
\begin{aligned}
\mathcal{L}_{n . m .}= & \frac{3}{2}\left[U \ln \left(\frac{U}{S_{0} \bar{S}_{0}}\right)-U+\frac{1}{3} U K\left(\bar{\phi}_{i} e^{q_{i} A} \phi_{i}\right)+\frac{2}{3} \hat{\xi} U(A+S+\bar{S})\right]_{D} \\
& +\left[S_{0}^{3} W\left(\phi_{i}\right)+\frac{1}{4} g\left(\phi_{i}\right) \mathcal{W W}\right]_{F} .
\end{aligned}
$$

Now $S$ induces an algebraic field equation, with solution $U=L$. But we instead eliminate $U$ by solving its field equation

$$
U=S_{0} \bar{S}_{0} \exp \left(-\frac{1}{3} K-\frac{2}{3} \hat{\xi}(A+S+\bar{S})\right)
$$

The resulting theory is

$$
\mathcal{L}_{\text {o.m. }}=-\frac{3}{2}\left[S_{0} \bar{S}_{0} \exp \left(-\frac{1}{3} K-\frac{2}{3} \hat{\xi}(A+S+\bar{S})\right)\right]_{D}+\left[S_{0}^{3} W+\frac{1}{4} g \mathcal{W} \mathcal{W}\right]_{F}
$$

and $S_{0}$ is now the chiral compensating multiplet of the old minimal formalism. Notice that when the linear $L$ is replaced by the real $U$, gauge invariance is obtained by assigning to $S$ a gauge variation such that $U$ is invariant. A combined gauge transformation of $S_{0}, S$ and (if $W$ is not zero and $R$-symmetric) $\phi_{i}$ allows then to eliminate $S$ which does not play any dynamical role, in complete correspondence with the globally supersymmetric case.

In old minimal theory (4.33), tensor calculus indicates that

$$
e^{-1} \mathcal{L}_{\text {o.m. }}=-\frac{1}{2} z_{0} \bar{z}_{0} \exp \left(-\frac{1}{3} K\right) R+\frac{1}{2} \hat{\xi} z_{0} \bar{z}_{0} e^{-K / 3} d+\ldots
$$

The dilatation fixing condition is now

$$
z_{0} \bar{z}_{0} \exp \left(-\frac{1}{3} K\right)=\frac{1}{\kappa^{2}}
$$

in the $S=0$ gauge, which, in the Wess-Zumino gauge, is also the lowest component of eq. (4.32), with $U_{\theta=0}=C=\kappa^{-2}$. In both theories (4.29) and (4.33) the FI coefficient is

$$
\xi=\frac{\hat{\xi}}{\kappa^{2}}
$$


With a superpotential breaking any $R$-symmetry, the natural supercurrent structure (4.9) always has a nonzero anomaly superfield $X$. The theory cannot be coupled to new minimal supergravity without breaking gauge invariance (4.26) which allows to gauge $S_{0}$ away. In the dual, old minimal version (4.33), one can for instance eliminate the $S$ field from the $D$-density using a Kähler transformation, or a holomorphic field redefinition of $S_{0}$. The result would be

$$
\mathcal{L}_{\text {o.m. }}=-\frac{3}{2}\left[S_{0} \bar{S}_{0} \exp \left(-\frac{1}{3} K-\frac{2}{3} \hat{\xi} A\right)\right]_{D}+\left[S_{0}^{3} e^{2 \hat{\xi} S} W+\frac{1}{4} g \mathcal{W W}\right]_{F} .
$$

The field equation for $S$ is now a constraint imposing $W=0$. Hence, further terms in $S$ are needed to make the field equations consistent. One should then consider a system with a supplementary dynamical chiral superfield $S$, i.e. with a non-minimal supergravity field content with $16_{B}+16_{F}$ off-shell fields. As observed by Komargodski and Seiberg [9], this is the natural supergravity formulation for the supercurrent structure with $X \neq 0 \neq \chi_{\alpha}$. This formulation of supergravity is usually named $16+16$ supergravity [20, 21, 22, 23].

One easily checks that in the global supersymmetry limit, the constraint induced by $S$ in the supergravity theory (4.37) vanishes as $\kappa^{2} \rightarrow 0$. Hence the natural supercurrent structure (4.9) is valid for an arbitrary superpotential.

It is clear that in the old or new minimal superconformal setups, one scale only is generated by the gauge-fixing of dilatations. Hence the resulting FI term is bound to be proportional to $\kappa^{-2}$ and the rigid limit $\kappa^{-2} \rightarrow \infty$ must be taken by requiring $\hat{\xi} \kappa^{-2}$ finite. In the $16+16$ supergravity setup however, with a linear $L$ and a chiral $S_{0}$, one can easily generate two independent scales at the price of $4_{B}+4_{F}$ supplementary dynamical fields. For instance,

$$
-\frac{3}{2}\left[S_{0} \bar{S}_{0} e^{-K / 3}\right]_{D}
$$

includes an Einstein term while

$$
\xi[L A]_{D}
$$

includes a "Fayet-Iliopoulos field" but no Einstein term. We can then associate the FI coefficient $\xi$ to the background value $\left\langle C_{L}\right\rangle$ while $S_{0}$ is gauge-fixed as in eq. (4.35) and generates then the Planck scale. Assuming $\left\langle C_{L}\right\rangle \ll \kappa^{-2}$ leads to an effective Fayet-Iliopoulos term with scale independent from the Planck scale in the global supersymmetry limit. 


\section{Conclusions}

We have reviewed the supercurrent structure of $N=1$ supersymmetric gauge theories in terms of the supercurrent and anomaly superfields. We have shown how superfield identities together with the field equations lead to a "natural" supercurrent structure for an arbitrary gauge-invariant Wess-Zumino model. We have pointed out that the supercurrent superfield is written in terms of off-shell superfields of the theory, but the supercurrent equation only holds on-shell. Then, the interpretation of the components of the supercurrent superfield as Noether currents requires the field equations. This is explicitly visible for the auxiliary field contribution to the energy-momentum tensor.

The natural supercurrent structure turns out to contain the Belinfante improved canonical (Noether) energy-momentum tensor and the Noether current for $U(1)_{\widetilde{R}}$ transformations, which leave chiral superfields inert. We have discussed two transformations of the natural supercurrent structure, which induce improvements of the supercurrent and energy-momentum tensor. The first one leads to a Ferrara-Zumino structure, which, for a generic theory, neither contains the new improved energy-momentum tensor of CCJ nor the Noether current of $U(1)_{R}$ transformations under which chiral superfields have a particular $R$-charge. The supercurrent structure containing these currents is obtained by a different transformation which in general retains the $16_{B}+16_{F}$ structure. Both transformations coincide for scale-invariant Kähler potentials.

Our unambiguous procedure to find supercurrent structures also applies to a supersymmetric gauge theory with a Fayet-Iliopoulos term, providing us with a natural gauge-invariant $16_{B}+16_{F}$ supercurrent structure. We have presented a possibility to transform the latter into a gauge-invariant Ferrara-Zumino structure. This relies on the introduction of a non-dynamical chiral superfield $S$, which does not change the field equations and the symmetries of the theory. It is just a purely formal device to obtain gauge-invariant Lagrangian superfields. We have then coupled the theory to supergravity and have pointed out that $S$ is naturally present due to a gauge transformation of the chiral multiplet $S_{0}$ in the superconformal formulation of new minimal supergravity. After performing the duality transformation to the old minimal formulation, we have found that the equations of motion for $S$ impose a vanishing superpotential. These complications vanish in the $\kappa^{2} \rightarrow 0$ limit to global supersymmetry, where $S$ can be always non-dynamical. For $R$-invariant superpotentials, they can be avoided by gauging the $R$-symmetry, which allows to gauge away $S$ and its equations of motion. For a generic superpotential this is not the case anymore and one is forced to introduce kinetic terms for $S$. This then turns $S$ from a mere formal tool to $4_{B}+4_{F}$ additional propagating fields, in agreement with the results of ref. [9]. 


\section{Acknowledgements}

We wish to thank Nicola Ambrosetti and Matthias Blau for useful conversations and contributions. J.-P. D. thanks the Simons Center for Geometry and Physics in Stony Brook for hospitality during part of this work. This research has been supported by the Swiss National Science Foundation.

\section{A Supersymmetric improvement transformation}

We take the following expansion of the supercurrent superfield $J_{\mu}$ :

$$
\begin{aligned}
J_{\mu}(x, \theta, \bar{\theta})= & \frac{8}{3} j_{\mu}(x)+\theta\left(S_{\mu}+2 \sqrt{2} \sigma_{\mu} \bar{\psi}_{X}\right)+\bar{\theta}\left(\bar{S}_{\mu}-2 \sqrt{2} \bar{\sigma}_{\mu} \psi_{X}\right) \\
& -2 i \theta \theta \partial_{\mu} \bar{x}+2 i \overline{\theta \theta} \partial_{\mu} x \\
& +\theta \sigma^{\nu} \bar{\theta}\left[8 T_{\mu \nu}-4 \eta_{\mu \nu} \operatorname{Re} f_{X}-\frac{1}{2} \epsilon_{\mu \nu \rho \sigma}\left(\frac{8}{3} \partial^{\rho} j^{\sigma}-F^{\rho \sigma}\right)\right] \\
& -\frac{i}{2} \theta \theta \bar{\theta}\left(\partial_{\nu} S_{\mu} \sigma^{\nu}+2 \sqrt{2} \bar{\sigma}_{\mu} \sigma^{\nu} \partial_{\nu} \bar{\psi}_{X}\right) \\
& +\frac{i}{2} \overline{\theta \theta} \theta\left(\sigma^{\nu} \partial_{\nu} \bar{S}_{\mu}+2 \sqrt{2} \sigma_{\mu} \bar{\sigma}^{\nu} \partial_{\nu} \psi_{X}\right) \\
& -\frac{2}{3} \theta \theta \overline{\theta \theta}\left(2 \partial_{\mu} \partial^{\nu} j_{\nu}-\square j_{\mu}\right) .
\end{aligned}
$$

The components of the superfields $X$ and $\chi_{\alpha}$ read

$$
\begin{aligned}
X= & x+\sqrt{2} \theta \psi_{X}-\theta \theta f_{X}-i \theta \sigma^{\mu} \bar{\theta} \partial_{\mu} x-\frac{i}{\sqrt{2}} \theta \theta \bar{\theta} \bar{\sigma}^{\mu} \partial_{\mu} \psi_{X}-\frac{1}{4} \theta \theta \overline{\theta \theta} \square x \\
\chi_{\alpha}= & -i \lambda_{\alpha}+\theta_{\alpha} D+\frac{i}{2}\left(\theta \sigma^{\mu} \bar{\sigma}^{\nu}\right)_{\alpha} F_{\mu \nu}-\theta \sigma^{\mu} \bar{\theta} \partial_{\mu} \lambda_{\alpha}-\theta \theta\left(\sigma^{\mu} \partial_{\mu} \bar{\lambda}\right)_{\alpha} \\
& -\frac{1}{2} \theta \theta\left(\sigma^{\mu} \bar{\theta}\right)_{\alpha}\left(\partial_{\nu} F_{\mu}^{\nu}-i \partial_{\mu} D\right)+\frac{i}{4} \theta \theta \overline{\theta \theta} \square \lambda_{\alpha}
\end{aligned}
$$

with $F_{\mu \nu}=-F_{\nu \mu}$ and $\partial_{[\mu} F_{\nu \rho]}=0$. If the real superfield $\mathcal{G}$ of the transformation (2.3) has the expansion

$$
\begin{aligned}
\mathcal{G}= & C_{g}+i \theta \chi_{g}-i \bar{\theta} \bar{\chi}_{g}+\theta \sigma^{\mu} \bar{\theta} v_{g \mu}+\frac{i}{2} \theta \theta\left(M_{g}+i N_{g}\right)-\frac{i}{2} \overline{\theta \theta}\left(M_{g}-i N_{g}\right) \\
& +i \theta \theta \bar{\theta}\left(\bar{\lambda}_{g}+\frac{i}{2} \partial_{\mu} \chi_{g} \sigma^{\mu}\right)-i \overline{\theta \theta} \theta\left(\lambda_{g}-\frac{i}{2} \sigma^{\mu} \partial_{\mu} \bar{\chi}_{g}\right)+\frac{1}{2} \theta \theta \overline{\theta \theta}\left(D_{g}-\frac{1}{2} \square C_{g}\right),
\end{aligned}
$$


then the components of the transformed superfields $\widetilde{J}_{\mu}, \widetilde{X}$ and $\widetilde{\chi}_{\alpha}$ read

$$
\begin{aligned}
\widetilde{j}_{\mu} & =j_{\mu}-3 v_{g \mu}, \\
\widetilde{S}_{\mu} & =S_{\mu}+8 \sigma_{[\mu} \bar{\sigma}_{\nu]} \partial^{\nu} \chi_{g}, \\
\widetilde{\psi}_{X} & =\psi_{X}+2 \sqrt{2} i \lambda_{g}+2 \sqrt{2} \sigma^{\mu} \partial_{\mu} \bar{\chi}_{g}, \\
\widetilde{x} & =x+2 i\left(M_{g}-i N_{g}\right), \\
\widetilde{T}_{\mu \nu} & =T_{\mu \nu}+\left(\partial_{\mu} \partial_{\nu}-\eta_{\mu \nu} \square\right) C_{g}, \\
\widetilde{f}_{X} & =f_{X}+2 D_{g}-2 \square C_{g}+2 i \partial_{\mu} v_{g}^{\mu}, \\
\widetilde{F}_{\mu \nu} & =F_{\mu \nu}-24 \partial_{[\mu} v_{g \nu]}, \\
\widetilde{\lambda} & =\lambda-12 \lambda_{g}, \\
\widetilde{D} & =D-12 D_{g} .
\end{aligned}
$$




\section{References}

[1] S. Ferrara and B. Zumino, Nucl. Phys. B 87 (1975) 207.

[2] M. F. Sohnius and P. C. West, Phys. Lett. B 105 (1981) 353.

[3] P. C. West, Introduction to supersymmetry and supergravity, World Scientific, Singapore (1990), 425 pages.

[4] M. Magro, I. Sachs and S. Wolf, Annals Phys. 298 (2002) 123 hep-th/0110131.

[5] S. M. Kuzenko, JHEP 1004 (2010) 022 [arXiv:1002.4932 [hep-th]]; Eur. Phys. J. C 71 (2011) 1513 arXiv:1008.1877 [hep-th]].

[6] T. T. Dumitrescu and N. Seiberg, JHEP 1107 (2011) 095 arXiv:1106.0031 [hepth]].

[7] S. J. Gates, M. T. Grisaru, M. Rocek and W. Siegel, Superspace or One Thousand and One Lessons in Supersymmetry, Benjamin/Cummings Publishing, Reading (1983) 548 pages; hep-th/0108200.

[8] I. L. Buchbinder and S. M. Kuzenko, Ideas and Methods of Supersymmetry and Supergravity: Or a Walk Through Superspace, Institute of Physics Publishing, Bristol (1995) 656 pages.

[9] Z. Komargodski and N. Seiberg, JHEP 1007 (2010) 017 arXiv:1002.2228 [hepth]].

[10] C. G. Callan, Jr., S. R. Coleman and R. Jackiw, Annals Phys. 59 (1970) 42;

S. R. Coleman and R. Jackiw, Annals Phys. 67 (1971) 552.

[11] N. Ambrosetti, D. Arnold, J.-P. Derendinger and J. Hartong, in preparation.

[12] T. E. Clark, O. Piguet and K. Sibold, Nucl. Phys. B 143 (1978) 445.

[13] T. Ortin, Gravity and Strings, Cambridge University Press (2004), 684 pages.

[14] E. Cremmer, S. Ferrara, L. Girardello and A. Van Proeyen, Nucl. Phys. B 212 (1983) 413.

[15] T. Kugo and S. Uehara, Nucl. Phys. B 222 (1983) 125.

[16] S. M. Kuzenko, Phys. Rev. D 81 (2010) 085036 [arXiv:0911.5190 [hep-th]].

[17] Z. Komargodski and N. Seiberg, JHEP 0906 (2009) 007 arXiv:0904.1159 [hepth]]. 
[18] K. R. Dienes and B. Thomas, Phys. Rev. D 81 (2010) 065023 arXiv:0911.0677 [hep-th]].

[19] C. M. Hull, A. Karlhede, U. Lindström and M. Roček, Nucl. Phys. B 266 (1986) 1.

[20] G. Girardi, R. Grimm, M. Muller and J. Wess, Phys. Lett. B 147 (1984) 81.

[21] W. Lang, J. Louis and B. A. Ovrut, Phys. Lett. B 158 (1985) 40.

[22] W. Siegel, Class. Quant. Grav. 3 (1986) L47.

[23] C. S. Aulakh, J. P. Derendinger and S. Ouvry, Phys. Lett. B 169 (1986) 201. 